\title{
A Study of Fronthaul Networks in CRANs - Requirements and Recent Advancements
}

\author{
Muhammad Waqar ${ }^{1}$, Ajung Kim ${ }^{2 *}$ and Peter K. Cho ${ }^{3}$ \\ ${ }^{12}$ Department of Optical Engineering, Sejong University, \\ Gwangjin-gu, 143-747 Seoul, South Korea \\ [e-mail: waqar@sju.ac.kr; akim@sejong.ac.kr] \\ ${ }^{3}$ Actus Networks Inc., Bangbae-dong, Seocho-gu, \\ 137-841, Seoul, South Korea \\ [e-mail: choho@actusnetworks.com] \\ *Corresponding author: Ajung Kim
}

Received January 8, 2018; revised April 15, 2018; accepted May 8, 2018;

published October 31, 2018

\begin{abstract}
One of the most innovative paradigms for the next-generation of wireless cellular networks is the cloud-radio access networks (C-RANs). In C-RANs, base station functions are distributed between the remote radio heads (RHHs) and base band unit (BBU) pool, and a communication link is defined between them which is referred as the fronthaul. This leveraging link is expected to reduce the CAPEX (capital expenditure) and OPEX (operating expense) of envisioned cellular architectures as well as improves the spectral and energy efficiencies, provides the high scalability, and efficient mobility management capabilities. The fronthaul link carries the baseband signals between the RRHs and BBU pool using the digital radio over fiber (RoF) based common public radio interface (CPRI). CPRI based optical links imposed stringent synchronization, latency and throughput requirements on the fronthaul. As a result, fronthaul becomes a hinder in commercial deployments of C-RANs and is seen as one of a major bottleneck for backbone networks. The optimization of fronthaul is still a challenging issue and requires further exploration at industrial and academic levels. This paper comprehensively summarized the current challenges and requirements of fronthaul networks, and discusses the recently proposed system architectures, virtualization techniques, key transport technologies and compression schemes to carry the time-sensitive traffic in fronthaul networks.
\end{abstract}

Keywords: 5G, Cloud radio access networks, CPRI, CRANs, fronthaul

This research was supported by MSIP/ IITP (No. R0127-15-1048) and by NRF (2018R1D1A1B07049877). 


\section{Introduction}

The demand for mobile data communications has been rapidly increased in last few years due to increased proliferation of smart devices and the number of users accessing bandwidth-hungry social and multimedia applications. A traffic forecast predicts that on average a cell phone user is expected to download data of around 1 terabytes annually in 2020 and $60 \%$ of this data will be video based [1]. Next-generation cellular networks are expected to provide ultra-high reliability, capacity, and connectivity to a huge number of smart devices and sensors to the internet, forming an internet of things (IoTs) [2]. The telecom industry is thus developing $5 \mathrm{G}$ technology with the objective of providing extensive data rates, high throughputs, extremely low latencies, large coverage, seamless connectivity, high energy and spectral efficiencies [3]. The mobile companies have limited options to upgrade existing infrastructure to satisfy the capacity demands, such as by increasing the number of cells [4] or integrating the advanced antenna technologies like Massive MIMO [5]. The densification of wireless networks with such approaches results in unavoidable inter-cell interferences [6], an increase of CAPEX (capital expenditures) and OPEX (operating expenses) of mobile network operators (MNOs) due to the deployment of more base stations (BSs) and power requirements to operate the BSs at cell sites. Whereas, average revenue per user (ARPU) decreases due to users expectations to pay less for more data usage [7]. Therefore, an optimal network architecture to deliver more capacity at affordable rates while maintaining the QoS (quality of service) are the requirements of next-generation 5G cellular systems.

Cloud radio access networks (C-RANs) has been recently proposed to alleviate the challenges of traditional radio access networks and to deliver high capacity to mobile users in a cost-effective manner [8]. In legacy radio access networks, the base station functions were located at a cell site in a unified unit. Whereas in C-RANs, base station functions are distributed between the dynamically distributed RRHs, and BBUs which are clustered as a BBU pool at a central cloud [9]. The RRH comprises low power antennas and carries all the radio frequency (RF) functions required for signal radiation at the cell site. The BBU is responsible for managing computational functions of baseband signals at a separate location. In such network architectures, cell sites are much simplified as well as power consumption and physical space requirements are reduced. The BBUs of several cell sites can interact with each other at lower latencies as co-located at a centralized cloud. The base station virtualization improves the resources pooling gain at a cloud and enables the deployment of a number of RRHs at a cell site as required by the network operator at minimum interferences by adapting the collaborative radio techniques such as cooperative multipoint (CoMP) [10]. Moreover, C-RANs are cost-effective as the number of base stations required at a cell site significantly reduced due to virtualization of baseband functionality at a central cloud rather than at different locations [11]. However, despite the potential advantages of C-RAN architecture, they come up with emerging implementation challenges. A fundamental challenge in the commercial deployment of C-RAN is a communication link between the RHHs and BBU pool, known as the fronthaul (FH), which is expected to meet the stringent latency and throughput requirements in order to transport the time-sensitive traffic between the RRHs and BBU pool [12]. An example of general C-RANs architecture is shown in Fig. 1.

The mobile network operators, telecom industry, and research community proposed a number of solutions to address the diverse challenges of C-RANs and fully exploit the advantages of base station virtualization for envisioned 5G cellular networks. To systematically show the 


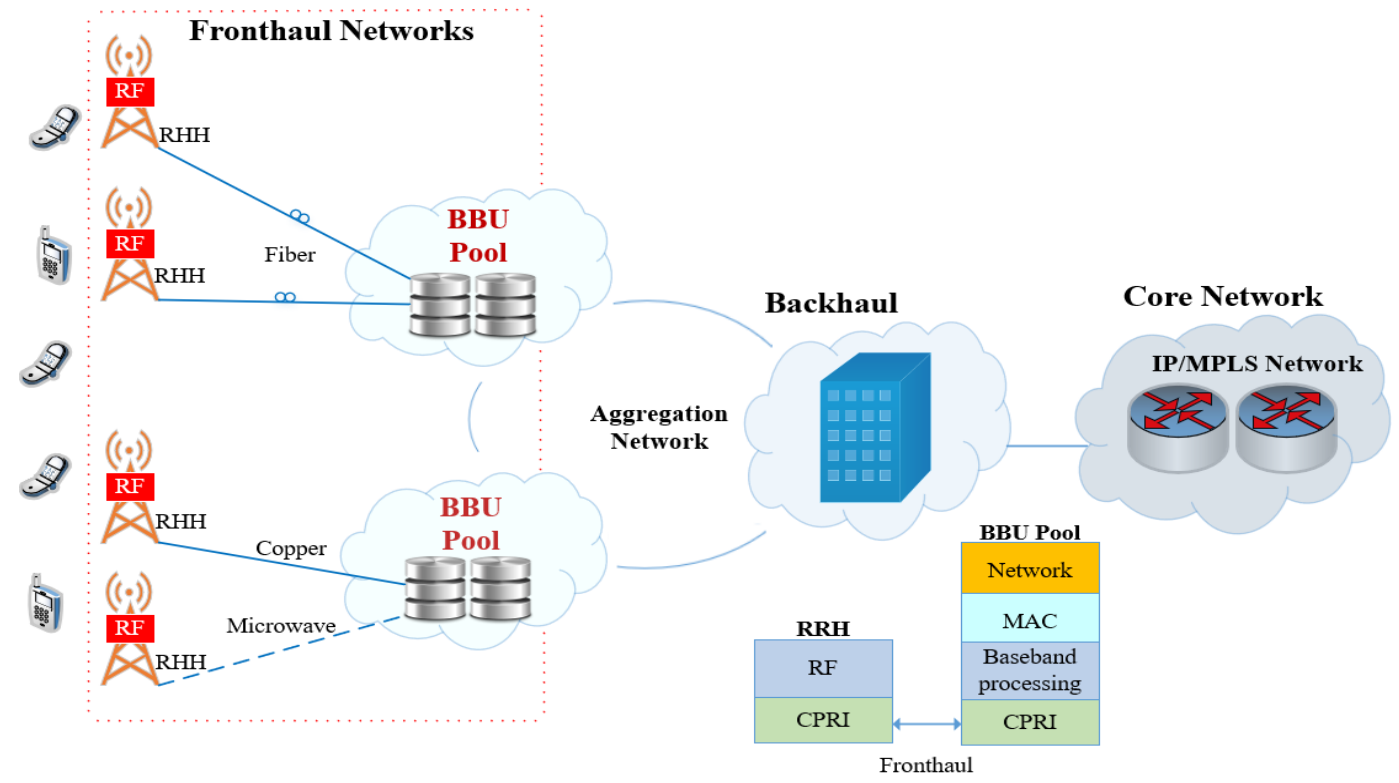

Fig. 1. The C-RANs general architecture

recent advancements and ongoing research activities, a comprehensive survey, especially on fronthaul networks can serve the researchers to understand the potential of fronthaul networks, exploit them further and develop more optimal solutions to make the C-RANs a reality in near future. The main contribution of this study can be summarized as follows:

(1) A comprehensive survey of fronthaul architectures proposed by academia and industry is presented, based on different functional splits. The trend lines between the achieved latencies and throughputs under different functional split options are discussed.

(2) The key virtualization techniques for next-generation fronthaul networks are comprehensively summarized. Particularly, advantages and role of software-defined fronthaul networks in C-RANs are surveyed.

(3) The research proposals and recent developments in transport technologies to carry the massive data over capacity constraint fronthaul link are elaborated.

(4) Recently proposed compression techniques to transport the time-sensitive traffic by efficiently utilizing the limited fronthaul bandwidth are summarized.

The rest of the study is organized as follows: Section 2 elaborates the main requirements of fronthaul networks. Section 3 presents a survey of fronthaul architectures in term of functional split proposed by the academia and industry. In Section 4, advantages of virtualization techniques and software-defined fronthaul networks are surveyed. In Section 5, recent developments in transport technologies including microwave, copper, and optical technologies to carry fronthaul traffic in a cost-effective manner are discussed. In Section 6, various compression schemes are presented. Section 7 summarizes the paper with some concluding remarks.

\section{Main Requirements of Fronthaul Networks}

Although C-RANs brings many advantages for 5G mobile cellular networks, they do come with their own challenges and requirements. The virtualization of BSs functionalities and 
physical separation of control and data plans in C-RANs imposes stringent latency and jitter requirements on the fronthaul link [12]. Fronthaul networks have bandwidth constraints which affect the performance gains of spectral and energy efficiencies. In addition, fronthaul is the most cost dominant component of C-RANs due to densely deployed RRHs and requirements of expensive fiber resources to connect huge numbers of RRHs to the BBU pool [13] [14]. Therefore, an optimal functional split between the RRHs and BBU pool is required that must meet the stringent delay and capacity requirements in a cost-efficient manner otherwise fronthaul would remain a bottleneck in commercial deployment of future 5G cloud radio access networks.

The 3GPP specification [15] defines the several functional splits between the RRHs or distributed unit (DU) and BBU pool or central unit (CU) to transport the baseband traffic over the fronthaul link. Each functional split has its own advantages and challenges. A high-level functional split has more efficient CoMP implementation capabilities, cost-effective and provides high multiplexing gains but imposes more stringent bandwidth, latency and jitter requirements on the fronthaul link. Whereas, a low-level functional split has flexible time synchronization and throughput requirements but requires efficient security mechanisms and lacks the implementation of CoMP services.

A network protocol is required to transport the baseband signals over a high-level FH that must satisfy the latency and jitter requirements without under or overutilization of limited bandwidth. For this purpose, the telecom industry has defined three leading protocols to transport fronthaul traffic, namely OBSAI (open base station architecture initiative) [16], CPRI (common public radio interface) [17], or ORI (open radio equipment interface) [18]. CPRI receives the highest attention and is adopted by many companies and MNOs because of its efficient mapping techniques and interoperability. CPRI is a layer 1 and layer 2 protocol, supports the bidirectional transmission of digital radio signals in form of quantized IQ (in-phase and quadrature-phase) samples at a constant bit rate along with the specifications of data and control planes. The CPRI defined the RF-PHY functional split between the RRHs and BBU pool [19] and supports a wide range of data rates from 614.4 Mbps (option 1) to 24.34 Gbps (option 10). Further, CPRI imposed stringent performance requirements on the FH and expecting to achieve one-way delay as low as $100 \mu$ s and jitter up to $65 \mathrm{~ns}$. Further, it requires precise time synchronization, special equipment to support an operating range of $10 \mathrm{~km}$ with a BER (bit error rate) of less than $10^{-12}$, and frequency deviations from the CPRI link to the radio base station less than $0.002 \mathrm{ppm}$ (parts per million) [20] such requirements can only be satisfied through an optical link [21], which further increases the FH deployment cost. In uplink transmissions from the numbers of RRH attached to a central BBU pool, an optical link can easily overwhelm due to continuous data transmission of CPRI protocol and a large number of attached users [22]. For instance, a LTE sector with $20 \mathrm{MHz}$ bandwidth, 2x2 MIMO configuration, and at CPRI line rate option 3 requires a data rate of 2.4 Gbps on the fronthaul link. Whereas, a 5G RRH equipped with 64 or 128 antennas, at CPRI line rate options 7 to 10 requires the FH bandwidth in the order of $100 \mathrm{Gbps}$, which increases the CAPEX due to expensive optical-switching equipment and large numbers of high capacity optical-interfaces between the densely deployed RRHs and BBU pool [22]. Therefore, compression schemes get attention as they are cost-effective and efficient in utilizing the limited bandwidth to transport the massive data. This requires the RRHs thus to perform the compression of received signals from all users before transmitting on the FH segment, and decoding functionalities at the BBU pool [23]. This solution introduces further technical challenges such as in densely deployed RRHs, user equipment receiving the signals from the multiple RRHs cause's significant interferences, and require the large-scale pre-coding and 
decoding schemes as well as precise channel estimation techniques at the BBU pool [24]. Thus, efficient compression, encoding, and decoding techniques are required for optical fronthaul networks to make them cost-effective. Recent advancements and solutions proposed to address the aforementioned fronthaul requirements and challenges in C-RANs are summarized in next sections.

\section{Fronthaul Architectures}

In $1 \mathrm{G}$ and $2 \mathrm{G}$ cellular systems, baseband processing and radio units were co-located as a single unit inside a base station. While in $3 \mathrm{G}$ and $4 \mathrm{G}$ cellular systems, the base station is divided into BBU and RRHs, which are located far away from each other. In the 5G systems, the functionality of BBUs are separated from the cell sites and relocated to a centralized BBU pool, where it serves multiple RRHs and the link between them is named as the fronthaul. Fig. 2 illustrates the evolution of BSs virtualization from the first generation to fifth generation mobile cellular networks.

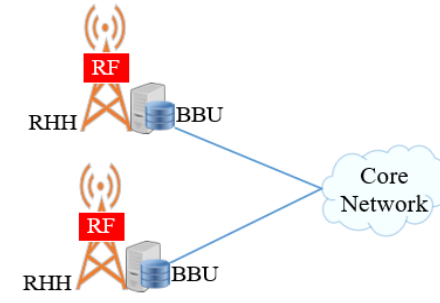

(a)

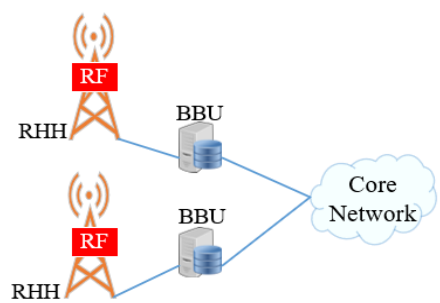

(b)

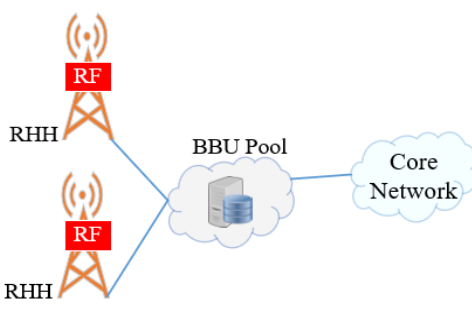

(c)

Fig. 2. Base station virtualization. (a) 1G/2G networks. (b) 3G/4G networks. (c) 5G-CRANs

\subsection{Proposed FH Network Designs by the Academia}

In legacy RAN architectures all base station functionalities are integrated into the BBU making RRH relatively inexpensive but in C-RANs to reduce the load of the transport network, base stations functionalities are split between the RRHs and BBU pool. The FH architecture can be categorized as fully centralized, partially centralized and hybrid centralized [12]. In fully centralized architecture, functions of the physical layer (PHY), data link layer (MAC: medium access control and RLC: radio link control), and network layer (PDCP: packet data convergence protocol) of BSs are moved into the BBU pool, as shown in Fig. 3 (a). Fully centralized architecture has the advantages of high data rates, scalability, maximum resources sharing, support to CoMP techniques, implementation of scheduling techniques and efficient multi-standard operation support as well as RRHs are under low load. In this format, traffic on the fronthaul link is very heavy because processing of IQ signals is moved at a central location and a RRH has to forward all traffic to BBU pool without any filtration and elimination. A 


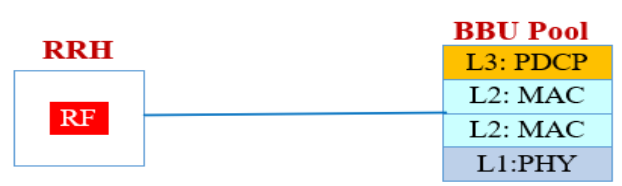

(a) Fully Centralized

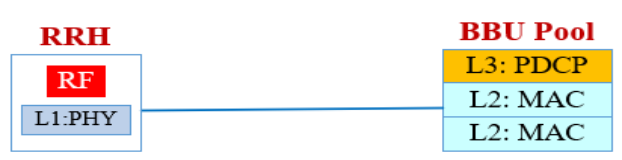

(b) Partially Centralized

Fig. 3. Functional split between RRH and BBU Pool. (a) Fully centralized. (b) Partially centralized

partially centralized scheme is shown in Fig. 3 (b) in which radio functions (RF) and PHY functions are shifted to a RRH and upper layer functions remain in the BBU pool. In this approach, the burden of a fronthaul link is overcome by moving the baseband functionality to the RRHs, but with the functional split of the MAC layer, multi-cell collaborative signal processing capabilities (e.g. CoMP) are significantly decreased in this architecture. This solution to split base station functionalities is less optimal as compared to fully centralized architecture that can support advanced features such as CoMP to deliver signal processing on Layer 1, 2 and 3 at a central BBU pool. A third case, known as hybrid centralized architecture in between the fully and partially centralization is a scheme in which only cell or user specific functions are migrated from the base station and integrated into a separate BBU pool. This brings advantages through resource sharing and a reduction in energy consumptions [12].

Several efforts have been made to choose an optimal functional split to achieve the desired performances in fronthaul networks. A trend line is derived in [25] to identify a tradeoff between the functional splits and their performances. Different centralization schemes in term of throughput and delays are evaluated in this study by varying the video traffic coming from a number of users over FH link towards the BBU pool. Fully centralization is suggested as the preferred functional split for the operators in term of user's QoS but at a high implementation cost. The partially centralization scheme reduces the cost but also the throughput of the network. The proposed fronthaul functional split [25] is classified into five types and their performances are shown in Table 1.

Table 1. Performances of different functional spits in fronthaul networks

\begin{tabular}{|c|c|c|c|}
\hline Functional Split & Latencies & $\begin{array}{c}\text { UL Throughput } \\
(\text { Mbps })\end{array}$ & $\begin{array}{c}\text { DL Throughput } \\
\text { (Mbps) }\end{array}$ \\
\hline \hline Split A - PDCP-RLC & $30 \mathrm{~ms}$ & 151 & 48 \\
\hline Split B - MAC & $6 \mathrm{~ms}$ & 151 & 49 \\
\hline Split C - MAC-PHY & $2 \mathrm{~ms}$ & 152 & 49 \\
\hline Split D - UE-Cell & $250 \mu \mathrm{s}$ & 1075 & 922 \\
\hline Split E - BBU-RF & $250 \mu \mathrm{s}$ & 2457.6 & 2457.6 \\
\hline
\end{tabular}

A novel concept of RANaaS (RAN-as-a-Service) to increase the flexibility of fronthaul architecture is proposed in [26]. Unlike traditional splitting schemes, on-demand and flexible cloud services such as congestion control, MAC and PHY layer management, remote radio resource monitoring and network management are proposed and evaluated under different network traffic loads. A study [27] analyzed the various functional splits and estimated the 
cost and performances achieved by the functional splits. To achieve high data rates with fully centralization approach between the RRHs and BBU pool a two-layered SoftAir solution is suggested in [28]. This scheme produces the high throughput under high traffic load but re-routing after handovers and paging accuracy are main challenges to be addressed yet.

Another comprehensive comparison of different functional splits and their impact on fronthaul and backhaul networks is analyzed in [29] and four functional split are categorized. Split A is a fully centralized approach. In Split B, received uplink signals are shifted to the frequency domain. In Split C, precoding, modulation, and equalization are locally performed, while forward error correction coding or decoding and higher layer functions are performed centrally. In Split D, all physical layer processing is locally performed while layer 2 and layer 3 functions are performed centrally. In partially centralization approach, results show that performance degrades in term of latency and throughput, as more functionalities are shifted toward the RRHs. Another concept of FH functional split is proposed in [30], which utilizes carrier aggregation (CA) among macro and small cell carriers. The physical layer and MAC layer functions are integrated into the RRHs and the control plane functions are performed in the BBU pool. This achieves high-performance gain by reducing the fronthaul burden and utilizing resources effectively. A fully centralized C-RAN architecture to serve hundreds of RRHs simultaneously and improve the network capacity is proposed in [31], as FH faces the huge amount of IQ sampling data when serving a large number of antennas at RRHs. To mitigate the huge data load while keeping the collaborative processing gain high, it is proposed to divide the physical functionalities into centralized and distributed parts. The BBU pool handles the precoding vectors and data symbols of users in the downlink. The RRH performs the precoding of user symbols and sends a radio signal over the link to reduce the network burden. Recently, a novel concept of fog computing- radio access network (FC-RAN) to solve the capacity and resource utilization issues at BBU pool is introduced [32]. Despite the innovative functional split design, this study requires more research on different aspects of fog computing concepts to make FC-RAN a new split option in future 5G radio access networks.

There is a tradeoff between the split type, throughput, and delays which a functional split can deliver in FH networks. A functional split closer to the BBU-RF (fully centralized) can deliver maximum throughput but at a high implementation cost and stringent latency requirements. The more functionalities are shifted from BBU to the RRHs (partially centralized), the cost and latency requirements are less but throughput also decreases. Therefore, more optimal functional split techniques and architectures are needed to make C-RANs a reality.

\subsection{Proposed FH Network Designs by Telecom Industry}

Many efforts have been made by the industry for practical deployment of fronthaul networks for envisioned C-RANs. In 2010, IBM first time proposed the C-RANs concept and now many companies demonstrated the fronthaul integration in LTE-Advanced cellular networks. DOCOMO [33] began developing a real experiment setup to upload data from UEs at 2 Gbps speed and Samsung is investigating to achieve the uplink data rate up to 7.5 Gbps on a stationary device and 1.2 Gbps when device moving at the speed of 100 kilometers per hour [34]. The recent developments and industry proposals to advance fronthaul networks are summarized in Table 2.

The mobile operators such as China Mobile and Korea Telecom are planning to deploy fronthaul networks commercially by 2020. Field trials are performed by the China Mobile and reduced the OPEX and CAPEX by 30\% and 53\% respectively for new cell sites as compared to D-RANs [44]. Korea Telecom together with Samsung is planning to deploy one thousand 
GPP based server at one BBU pool, each server can manage 144 base stations. Also proposed a CCC (cloud communications center) architecture for C-RANs deployments [45]. However, achieving the desired capacity and latency requirements of FH are under investigation.

Table 2. Industrial proposals for fronthaul advancements

\begin{tabular}{|c|c|c|c|}
\hline Year & $\begin{array}{l}\text { Corpor- } \\
\text { ate }\end{array}$ & $\begin{array}{c}\text { Proposed } \\
\text { Technology }\end{array}$ & Contribution \\
\hline 2010 & $\begin{array}{l}\text { China } \\
\text { Mobile } \\
\text { [14] }\end{array}$ & $\begin{array}{l}\text { FH functional } \\
\text { split }\end{array}$ & $\begin{array}{l}\text { A detailed C-RAN architecture is presented, Analyzed the } \\
\text { technology trends and feasibility of C-RANs functional } \\
\text { split with experimentations }\end{array}$ \\
\hline 2010 & $\begin{array}{c}\text { IBM } \\
{[35]}\end{array}$ & $\begin{array}{c}\text { Wireless } \\
\text { network cloud }\end{array}$ & $\begin{array}{l}\text { Firstly suggested the concept of fronthaul networks. } \\
\text { Decreases networking cost and brings the flexibility in } \\
\text { RANs by separating hardware and software services }\end{array}$ \\
\hline 2011 & $\begin{array}{l}\text { ZTE } \\
{[36]}\end{array}$ & $\begin{array}{l}\text { Color fiber } \\
\text { connection, } \\
\text { WDM/OTN }\end{array}$ & $\begin{array}{l}\text { Introduces the enhanced and color fiber connections for } \\
\text { fronthaul transport network, alleviates the fiber scarcity in } \\
\text { order to fulfill the fronthaul requirements with less number } \\
\text { of optical cables }\end{array}$ \\
\hline $\begin{array}{l}2011 \\
2013\end{array}$ & $\begin{array}{l}\text { Alcatel-L } \\
\text { ucent } \\
\text { [37] [38] }\end{array}$ & $\begin{array}{l}\text { Virtualized cell } \\
\text { sites, Light } \\
\text { Radio solution }\end{array}$ & $\begin{array}{l}\text { Reduces the overall resources processing without } \\
\text { degrading the system performances. All-in-one small } \\
\text { footprint to host all BBUs in a small box is proposed which } \\
\text { reduces the cooling and rental cost of the FH networks }\end{array}$ \\
\hline 2014 & $\begin{array}{l}\text { Nokia } \\
\text { Siemens } \\
\text { [39] [40] }\end{array}$ & $\begin{array}{l}\text { Liquid Radio, } \\
\text { WCDMA } \\
\text { software suite }\end{array}$ & $\begin{array}{l}\text { Improves the network utilization by adopting centralized } \\
\text { FH architecture. Precisely manages unpredictable traffic } \\
\text { demands and reduces the cost of ownership to facilitates } \\
\text { the FH demands. }\end{array}$ \\
\hline 2015 & $\begin{array}{c}\text { Ericsson } \\
\text { [41] }\end{array}$ & $\begin{array}{l}\text { Automating } \\
\text { network service } \\
\text { lifecycle } \\
\text { management } \\
\end{array}$ & $\begin{array}{l}\text { Resource prioritization is implemented for FH. The latency } \\
\text { of mission-critical traffic remain same even network } \\
\text { reaches maximum congestion level and overloaded. In a } \\
\text { demonstration speed of } 5 \text { Gbps is successfully achieved }\end{array}$ \\
\hline 2016 & $\begin{array}{l}\text { OSA } \\
{[42]}\end{array}$ & $\begin{array}{l}\text { Combined the } \\
\text { SDN, NFV, and } \\
\text { OpenStack with } \\
\text { the open source } \\
\text { software }\end{array}$ & $\begin{array}{l}\text { Implemented a central cloud network using new entities } \\
\text { and packet switching design on Linux-based computing } \\
\text { equipment to achieve high capacity and reduce the } \\
\text { stringent latency and jitter requirements of fronthaul } \\
\text { networks }\end{array}$ \\
\hline 2017 & $\begin{array}{c}\text { E-Blink } \\
\text { [43] }\end{array}$ & $\begin{array}{l}\text { Wireless } \\
\text { fronthaul } \\
\text { systems }\end{array}$ & $\begin{array}{l}\text { Providing the capacity of } 7.5 \text { Gbps from } 1 \text { to } 3 \text { sectors up to } \\
400 \mathrm{~m} \text { line of sight communication based on LTE and } \\
\text { UMTS frequency bands to comply the fronthaul demands }\end{array}$ \\
\hline
\end{tabular}

\section{Virtualizations in Fronthaul Networks}

This section presents advantages of virtualization and recent advancements proposed to virtualize the fronthaul networks and computing resources at BBU cloud. The virtualization in networks provides flexibility to convert the physical networking model into the program-able and centralized management environment to reduce the cost and efficiently utilize the resources. An existing infrastructure is upgraded to support new applications without hardware up-gradations. SDNs (software-defined networks) and NFV (network function virtualization) are two important concepts to implement virtualization in networks and allow 
services to be automatically configured and executed without increasing the network cost [46] because high implementation cost (CAPEX) is a major challenge of FHs in C-RANs.

Like traditional mobile networks, an SDN-based FH architecture mainly consists of three parts, a software controller, southbound and northbound parts. The software controller is responsible for controlling APIs, network resources, network operating system and maintaining a global view of the network. The southbound part provides an interface using the protocols such as OpenFlow [47] or OpenDaylight [48] between the software controllers and SDN infrastructur-es. The northbound part provides an interface between the controller and SDN applications.

To take the advantages of SDNs and make the fronthaul segment more virtualized and dynamic, an SDF (software defined fronthaul) is introduced in [49]. An SDF is achieved by splitting the radio access network functionality into two parts, one of which is processed at the base station while the other is executed in a central processing BBU unit. The basic idea of using an SDF is to allow the decoupling of a network into data plane (to perform forwarding) and control plane (to perform configuration and management) and defining the mechanism between the two planes using a well-defined interface. The functions of the control plane are centralized in a software-defined fronthaul, which provides APIs (application program interfaces) to the operator for the given technology. Using software-based controllers, the operator has the options to instruct data plane nodes such as routers to perform data forwarding functions according to traffic requirements. To observe the user profiles and traffic load, a resource manager is needed with a high capacity switch for real-time reconfigurations. The resource manager performs two functions, firstly determining the total number of BBUs which are transmitting frames and defining a mapping method between the BBU pool and RRHs, and secondly allocates the processing cores to each BBU. After mapping, a resource manager sets the switching element to unicast/multicast mode and activate the specific output ports to intended recipient RRHs. The BBU pool can serve a number of small RRHs with this scheme, ensuring scalability, reliability and high capacity.

An optimized framework of FluidNet [50] is proposed to improve the performance of C-RANs by intelligently reconfiguring the fronthaul between the RRHs and the BBU pool. FluidNet is tested in a partially centralized C-RANs, where better performance is achieved by reducing the load of RRHs. Results with a test bed consisting of a BBU pool and six RRHs in WiMAX show that FluidNet strategies and capability of adaptive reconfiguration achieves 50\% impro-vement in data throughputs and reduces computing resource utilization in the BBU pool by $50 \%$ compared to legacy schemes. A software-defined wireless network controller (SDWNC) is introduced in [51], which is located within the BBU cloud. It acts as a supervisor and administrator node to control and apply the self-healing procedures to all cell sites based on the software defined-wireless networks. Due to the SDWNC location in the cloud, an optimal decision is made in a very short time to recover the fronthaul failures. It activates the self-healing radio procedures for all cell sites in an area affected by a failed cell site and deactivates the process after the failure is repaired. The proposed method is verified with simulations which indicate that at least $20 \%$ fronthaul failure rates can be recovered for any network topology. In [52], the author proposed a new packetized version of fronthaul traffic in $5 \mathrm{G}$ cross-haul architecture to integrate existing and new fronthaul interfaces and technologies. Unlike existing techniques which only targeted the dynamic reconfiguration of the cognitive control planes and small cells, by joining the optical and wireless backhaul and fronthaul architectures, a new design of unified data and control plane is proposed for all types of fronthaul and backhaul network by applying NFV and SDN principles. 
A case study [53] analyzed the long-distance optical fiber based FH networks by connecting the RRHs to metro-core central offices (MCCO) at minimum latencies. This study proposed the flex-grid optical transport networks to handle the low-cost wireless BBUs to transmit the OFDM (orthogonal frequency-division multiplexing) signals up to $40 \mathrm{~km}$ to provide a long-distance reliable connectivity in the fronthaul and backbone networks. RRHs are connected to the wireless BBUs which are attached to the MCCO via optical fiber. The experimental result shows that hardware virtualization using NFV techniques facilitate the efficient long-distance communication and significantly reduces the CAPEX of the FH networks. In [54], two SDN based techniques namely, vertical convergence and horizontal convergence are introduced to transport the traffic and achieve the flexibility in optical FH networks. In vertical convergence, multiple layers of network stacks are converged such as optical and radio domains. Whereas, in horizontal convergence, BBUs and networking resources are congregated. An OpenDay-light based controller is suggested to implement the flexible grid optical networks using the vertical and horizontal converges. This study shows that elastic lightpath provision as a C-RAN service can be achieved by adapting the horizontal and vertical convergences.

An SDN based optical access network is experimentally demonstrated in [55] to achieve the resources virtualization between the RRHs and BBU pool. The result shows that RRH-BBU mapping using the software-based bandwidth allocation techniques improves the CoMP performance and utilizes the cloud resources more efficiently. The study [56] proposes a mechanism to associate the several RRHs to a single BBU pool. The proposed mechanism is emulated on the TWDM optical fronthaul network under different cell conditions. The author claims that efficient CoMP services, reduced data transmissions, and low latencies are successfully achieved with proposed schemes. A concept of BBU aggregation to multiplex the IQ samples of RRHs in time-sensitive fronthaul networks by improving the energy efficiencies is proposed [57] and evaluated with different cell sites including heterogeneous, micro, macro and pico cells. Numerical result shows that energy consumptions can be minimized especially in small cell scenarios by adapting the flexible BBU aggregation mechanisms.

Although SDN techniques of the fronthaul are yet to be defined in the standard, the concept of introducing software layer above the control plane can assist a lot fronthaul architecture by increasing operability in using different vendor's software and hardware components. SDF will enable the operators to manage fronthaul traffic loads and capacity virtually without changing the hardware components. However, a combination of proposed SDN and NFV techniques such as FluidNet, SDO, wireless BBUs, and SDWNC can be standardized and coupled together to achieve the self-configuration, load balancing, auto-failure detection and auto-healing features in fronthaul networks.

\section{Transport Technologies for Fronthaul Traffic}

In C-RAN architecture, the load on the FH link is 50 times higher than the backhaul link, which when coupled with the significant traffic demands make the fronthaul interface more complicated. For different network operators, the choice of adopting a transport technology for a FH segment depends on whether FH is deployed on top of legacy infrastructure or started from scratch. Therefore, based on the operator choice and network requirements, there are three options for a fronthaul transport link i.e. microwave based, Ethernet based, or optical based. A selected FH transport link should be capable of satisfying the stringent delay, jitter and throughput requirements in a cost-effective manner. This section discusses the potential of 
currently available technologies and recent developments to carry the massive and time-sensitive traffic in capacity constraint fronthaul networks.

\subsection{Microwave-Based Fronthaul Link}

Typically, microwave can offer $100 \mathrm{Mbps}$ to 1 Gbps data rate for long distances and up to 1 Gbps for a distance of $1.5 \mathrm{~km}$ [58]. A study [59] proposes to apply E-band microwave as a transmission medium for FH interfaces at $70 / 80 \mathrm{GHz}$. In the laboratory conditions, up to 2.5 Gbps transmission rate has been successfully achieved for a distance of 1 to $2 \mathrm{~km}$ while maintaining the delays in the range of few microseconds. Wireless fronthaul equipment has been developed by [43] to achieve the data rates up to $7.5 \mathrm{Gbps}$ within the $70 \mathrm{MHz}$ of spectrum for one to three sector of LTE. In [60] Wi-Fi standard IEEE 802.11ad has been proposed as a potential medium for wireless fronthaul networks that can achieve theoretical throughput up to 7 Gbps in the laboratory setup but the range of Wi-Fi is very short as compared to microwave and limited to indoor communications only.

\subsection{Ethernet-Based Fronthaul Link}

One of a hurdle in commercial deployment of the fronthaul is a high implementation cost of the optical link. In an effort to make fronthaul interfaces cost efficient while achieving higher throughputs, many proposals have been recently suggested which are based on Ethernet switching equipment, such as CPRI over Ethernet (CoE) [61-67]. Because Ethernet is a mature technology, highly scalable, up-gradable to higher data rates, inexpensive due to its ubiquitous deployment and even for large FH networks, the configuration of Ethernet switches is feasible but delay and jitter are the major challenges.

IEEE suggested two enhancements, i.e. IEEE 802.1Qbu [61], referred as Ethernet preemption, and IEEE 802.1QBv [62], referred as Ethernet scheduled traffic, to carry time-sensitive traffic in packet-switched networks. These solutions are not proposed directly for $\mathrm{CoE}$ enhancements but are strongly relevant and applicable to CoEs. The studies [63-64] thus brings attention to utilize the IEEE 802.1Qbu and IEEE 802.1Qbv to improve the Ethernet capability to meet the fronthaul demands in terms of delays and jitter. This study shows that standard Ethernet cannot meet the FH delay and jitter requirements but with the adoption of IEEE 802.1Qbu standard, delay within the acceptable range can be achieved. On the other hand, Ethernet with IEEE 802.1Qbv is efficient in reducing the jitter in some cases, but not in all, which means that coupling preemption and scheduling techniques together can be utilized to achieve desired delay and jitter values in Ethernet-based FH networks. Another attempt to investigate the CoE networks to evaluate the FH demands was carried out recently [65]. A Comb-Fitting (C-FIT) algorithm that is based on traffic scheduling (IEEE 802.1Qbv) is employed to reduce the jitter. Authors experimentally proved that packet-switched Ethernet technology is credible to transport CPRI traffic between the multiple RRHs and BBU pool while maintaining the delays and jitter within the fronthaul requirements under certain conditions such as at a low traffic load and low CPRI line rates referred as LER (load to Ethernet ratio). This study shows that by adopting the traffic scheduling techniques in fronthaul networks, delays within $100 \mu$ s and jitter within 65 ns can be achieved in 10 Gbps Ethernet switching networks.

The IEEE 1904.3 working group [66] is investigating on standardizing the encapsulation techniques and transport protocols to carry the radio samples over the Ethernet-based fronthaul networks. The objective of this standards also includes a transport mechanism to carry a number of radio streams simultaneously over Ethernet link by adapting the radio over Ethernet (RoE) techniques. IEEE P802.1CM working group [67] is currently striving to 
develop the new profiles, features, procedures and standardize the protocols for building a packet-switched network to transport FH streams without much delays over Ethernet link. Although, the concept of $\mathrm{CoE}$ or RoE has been clearly defined but more work is required to optimize the delays and jitter requirements to maximize the benefits of Ethernet based fronthaul in C-RANs.

\subsection{Optical-Based Fronthaul Link}

Fronthaul transport link is expected to meet both latency and capacity requirements, thus optical fiber is the most appropriate option due to its high capacity and capabilities to ensure the time synchronization between the traffic flows. Several studies evaluated the optical-fronthaul networks to maximize the RRHs per BBU, distances a capacity constraint link can support without degrading the performance and to reduce the number of fiber resources.

A TDM based Mobile-PON (passive optical networks) scheduling algorithm and a PHY funct-ional split between the DU and CU is proposed in [68] to transport the CPRI traffic over the FH segment. This study shows that bandwidth utilization can be improved 10 times as compared to legacy PON transmissions with the proposed techniques. The study [69] proposed an optical ring topology to support CPRI specifications and fulfill the fronthaul traffic demands. In [70] to eliminate the serialization delays in optical bridged networks, a novel route optimization technique is proposed to transport the traffic of RRHs at guaranteed QoS. The simulation result shows that up to hundred RRHs can be attached to a BBU pool while fulfilling the fronthaul QoS requirements. In [71], colored fiber and enhanced fiber connect-ions are proposed to build the fronthaul networks to achieve 10 Mbps to $1 \mathrm{Gbps}$ data rates in dense areas at low cost.

A non-hierarchical WDM solution for fronthaul networks is discussed in [72]. This work shows that such proposed solutions can improve the network transparency and costs especially in clustered based base stations. The author suggested to utilize PONs or metro rings to support the CPRI traffic in fronthaul networks and implemented a point-to-point WDM setup to show 48 CPRI links per fiber successfully. Coarse-WDM (wavelength division multiplexing)-PON is a cost-effective solution but support limited channels. Whereas Dense-WDM is a preferred solution that can support dynamic channel allocation techniques and up to tens of Gbps data rates with NG-PON (next generation-PON) technology but DWDM is cost ineffective. However, E-PON (Ethernet-PON) and GE-PON (Gigabit Ethernet-PON) are two cost effec-tive alternative options to achieve data rates from 1 Gbps and 10 Gbps respectively.

ZTE proposed [73] the enhanced fiber connection and colored fiber connections as a feasible solution for widespread C-RAN deployments. This solution connects 18 cascaded RRHs with one pair of fiber along with the utilization of mature DWDM, OAM (operations, admini-stration, and maintenance) and FEC (forward error correction) to fulfill the requirements of LTE. A high-capacity optical FH network architecture is implemented in [74] based on wavelength sharing in which several cell sites share a single DWDM channel rather than several channels. On a single wavelength, four $20 \mathrm{MHz}$ LTE, 16-QAM signals at a spacing of $100 \mathrm{MHz}$ can be multiplexed to increase the spectral efficiency in the FH network. This work integrated the SCM (sub carrier multiplexing) with DWDM for downlink transmission and utilizes ultra-dense WDM allocation over DWDM at wavelength spacing of $1 \mathrm{GHz}$. With proposed uplink and downlink techniques, the author claims that a high capacity and spectrally efficient optical-FH networks can be realized. A transport network solution using DWDM is established in [75] that reduces network complexity, improves load balancing 
and provide redundant paths along with auto configurations in the network. A study [76] proposed the techniques to recuse the existing PON infrastructure to be a bearer for IQ traffic between the RRHs and BBUs using DWDM-PON and coherent optical OFDM with small channel spacing to reduce the cost of the network. An OFDM-WDM architecture based on UniPON is proposed and evaluated in [77]. It is shown that such networks can carry 14 wavelengths per fiber and reduces the cost because of resource sharing. A recent work [78] validates the multi-core fiber (MCF) suitability for fronthaul networks. This study shows that worst IC-XT (intercore-crosstalk) reaches up to $9.6 \mathrm{~dB}$. Experiments are performed by aggregating three LTE-A carrier's transmission with radio over fiber technology. The author proposed to apply MIMO-MCF mitigation schemes to achieve high throughput and better performances than SISO (single input single output) transmission.

A concept of photonics-aided amplification is proposed in [79] to enhance the SNR, improve the throughput and mitigate the cell edge user interferences for next-generation FH networks. The experimental results show that throughput of FH networks are doubled and BER is improved by $2.5 \mathrm{~dB}$ with the utilization of photonics-aided amplification. A feasibility of utilizing RoF with WDM is demonstrated in [80]. An experimental setup for sharing cloud-based RoF in small cell network is developed for two operator's coexistences to deploy a versatile, power-efficient and cost-efficient fronthaul network. Table 4 summarized the recent developments and proposals to utilize the optical fiber as a transport technology for FH.

Table 4. Optical transport technologies proposed for FH networks

\begin{tabular}{|c|l|l|}
\hline $\begin{array}{c}\text { Optical } \\
\text { Technology }\end{array}$ & \multicolumn{1}{|c|}{ Contribution } & \multicolumn{1}{c|}{ Limitations } \\
\hline \hline $\begin{array}{c}\text { TDM-PON } \\
\text { [68] [69] }\end{array}$ & $\begin{array}{l}\text { Improves the network performance and } \\
\text { FH bandwidth 10 times for CPRI traffic to } \\
\text { achieve the cost-benefits of PON networks }\end{array}$ & $\begin{array}{l}\text { Increases the complexity of FH } \\
\text { interface and overhead at } \\
\text { switches due to scheduling }\end{array}$ \\
\hline $\begin{array}{c}\text { TDD-PON } \\
\text { [70] }\end{array}$ & $\begin{array}{l}\text { Transport the fronthaul traffic of 100 } \\
\text { RRHs in a bridged network at guaranteed } \\
\text { QoS }\end{array}$ & $\begin{array}{l}\text { Proposed an intelligent optical } \\
\text { switch architecture which is } \\
\text { difficult to design }\end{array}$ \\
\hline $\begin{array}{c}\text { WDN-OTN } \\
\text { [71] }\end{array}$ & $\begin{array}{l}\text { Provide solutions to connect RRHs with } \\
\text { BBU in dense areas to achieve speeds } \\
\text { from 10 Mbps to 1 Gbps }\end{array}$ & $\begin{array}{l}\text { Utilization of WDN-OTN and } \\
\text { colored fiber with connections } \\
\text { increases the deployment cost }\end{array}$ \\
\hline $\begin{array}{c}\text { Non-hierarchical } \\
\text { and point-to-point }\end{array}$ & $\begin{array}{l}\text { Improve the transparency and network } \\
\text { cost especially in centralized base stations. } \\
\text { WDM }\end{array}$ & $\begin{array}{l}\text { Increases installation cost. } \\
\text { Traffic monitoring and load } \\
\text { balancing is required to retain } \\
\text { per optical fiber }\end{array}$ \\
t72] network performance
\end{tabular}




\begin{tabular}{|c|l|l|}
\hline & low inter-core crosstalk & network \\
\hline $\begin{array}{c}\text { Photonics-aided } \\
\text { amplification } \\
{[79]}\end{array}$ & $\begin{array}{l}\text { Improve throughput, reduces cell edge } \\
\text { user interferences and SNR. BER is } \\
\text { reduced 2.5 dB times }\end{array}$ & $\begin{array}{l}\text { Require complex setup for } \\
\text { fiber transmission that results } \\
\text { in high CAPEX }\end{array}$ \\
\hline $\begin{array}{c}\text { Cloud RoF-WDM } \\
{[80]}\end{array}$ & $\begin{array}{l}\text { Provide operators coexistence, reduces } \\
\text { cost and power utilization }\end{array}$ & $\begin{array}{l}\text { Require extensive control and } \\
\text { management protocols at cloud }\end{array}$ \\
\hline
\end{tabular}

To support CPRI traffic over the optical-based fronthaul networks various switching equipment has been developed by telecommunication industry. The study [81] developed a CPRI Bundle to validate and test the CPRI protocols using programmable high-speed FPGA based serial modules. A module is introduced in [82] to reduce the time and cost of testing RRHs and BBUs communication based on RF and CPRI protocols with auto detecting and auto-configuration feature to reduce the errors and ease the testing. A CPRI MUX is developed by [40] to aggregate, encapsulates, and transport the traffic of several RRHs over CWDM and DWDM optical links. A Fat-Tree dynamic routing switch [44] is developed by the China Mobile to support more than 10k carriers per BBU pool. The performance of CPRI over OTN has been analyzed by [83] using the OTN processors as a switch to map client signals into ODU (optical channel data unit) [84].

To conclude, currently available optical fiber technologies especially CWDM-PON and DWDM-PON are the preferred possible solutions for deployment of practical FH networks to achieve throughput up to tens of Gbps but at a high deployment and equipment cost while E-PON and GE-PON are more cost-effective alternatives to achieve data rates up to $10 \mathrm{Gbps}$ at latencies in few microseconds. Ethernet is a cost-effective solution to carry CPRI traffic but requires the advanced algorithms like traffic scheduling to route the CPRI streams at low latencies in order to meet the fronthaul requirements. Microwave technologies can be utilized for short distance located RRHs from BBU pool at a low traffic load to support data rates up to 1 Gbps.

\section{Compression Techniques for Fronthaul Interface}

A standard solution for fronthaul transport network can be optical fiber to carry the massive streams of CRPI in form of IQ samples between the RRHs and BBU pool. In C-RANs, even under normal traffic conditions bandwidth required to carry IQ samples is high. For instance, as investigated in [83], an LTE macro-base station with limited functionality requires the data rates that exceed the $100 \mathrm{Gbps}$. The problem is more dominant in the case of densely deployed RRHs, in which a user equipment with few Mbps of capacity can easily overwhelm the 10 Gbps of optical capacity even under moderate traffic conditions. Therefore, efficient techniques are needed to transport the massive FH data over a capacity-constrained link such as the use of non-linear quantization, reducing signal sampling rate, and frequency sub-carrier compression or compression of IQ data [14]. IQ data compression is the most preferred solution proposed so far due to the fact that it offers efficient capacity utilization and improves the network performance as compared to non-linear quantization that increases the interface complexity between BBU and RRHs and signals sampling causes performance degradation [14]. A network operator needs to either utilize compression schemes or own substantial amount of fiber to provide the required capacity. However, by just applying the compression techniques in uplink and downlink, the cost of fiber resources and optical equipment can be saved. Therefore, IQ compression techniques get much attention of the researcher community and industry. Recently proposed compression schemes to improve the utilization of fronthaul 
bandwidth while retaining the 3GPP requirements of EVM as low as $8 \%$ for 64 QAM and BER $1.9 \times 10^{-3}$ respectively [85], minimizing the SNR, maximizing the weighted sum and compression ratios are summarized in this section.

\subsection{Uplink Compression}

For uplink compression, RRHs compress the received baseband signals and transmit them to the BBU pool where decompression and decoding on received signals are performed. To achieve the low BER and SNR, a concept of CCT (charnes cooper transformation) is suggested [86] with difference-of-convex programming to devise an algorithm to reduce localization errors of the single RRH traffic with the worst-case scenario in capacity constrained FH. Distributed compressive sensing and joint recovery techniques are applied for compression of IQ data and end-to-end signal recovery algorithm is proposed [87]. A tradeoff between the distributed fronthaul load and uplink capacity is analyzed to make the proposed technique applicable to practical deployments. A comparison of per active user throughput on a fronthaul link as a function of the number of bits per link is performed for various compression schemes with variable active users by maintaining the transmitted SNR to $20 \mathrm{~dB}$. It is observed that per active user throughput is supposed to increase as the FH load increases. It is also induced that the proposed scheme shows performances as high as those of the ideal Genie-aided ZF, and as the fronthaul load increases then the probability of RIP (restricted isometry property) and of correctly detecting active users is also increases. Hence, results provide the foundation to apply carrier sensing schemes on uplinks.

Unlike the previous schemes in which RRHs and the BBU pool are directly connected, a multi-hop fronthaul scenario [88] is analyzed, in which RRHs communicates with BBU through intermediate RRHs. In this configuration, multiplexing and forwarding methods may become less feasible with densely deployed RRHs. The DPR (decompress process and recompress) scheme is proposed to solve this problem, in which every RRH first decompress the signal and applies linear processing to decompress the received signals. In this work, the advantages of in-network processing are explained with a comparison of the performance of multi-hop scheme and DPR scheme. Uplink compression based on uniform quantization and OFDM is proposed by [89] in which each UE is allocated orthogonal sub-carriers for transmission. This maximizes the system throughput with quantization and wireless power control. The results show that significant performance gain is achieved with the proposed scheme, which reveals the possibility of supporting dense RRHs deployment with compression techniques.

The SCF (spatial compression and forward) technique is proposed in [90] to manage and control traffic from the RRHs for uplink transmissions. The RRHs firs perform linear spatial filtering to remove noise in this scheme then compares the signal from the UEs to a minimum dimension, performs uniform scalar quantization on outputs, and then forwards the quantized bits to the BBU pool for decoding. This scheme with a BBU's joint resource allocation on radio and FH links maximizes the minimum SINR (signal-to-interference-plus-noise ratio) of users and reduced the burden on the links.

\subsection{Downlink Compression}

The downlink signals go through channel coding, precoding, separate or joint compression at the BBU pool, and decompression at the RRHs before transmission to a particular user in the network. An efficient compression scheme is proposed by Alcatel-Lucent Bell Labs that decreases the FH interface traffic to $8 \mathrm{Gbps}$ from $18 \mathrm{Gbps}$ at $44 \%$ compression ratio [40]. An 
adaptive compression algorithm is utilized [91] to remove the frequency redundancy and achieve the compression ratio $25 \%$ to $50 \%$ and EVM from $0.5 \%$ to $8 \%$ for LTE signal over CPRI interface. A joint precoding design and compression for multi-cluster C-RANs are studied in [92]. The weighted sum rate is maximized between multiple clusters with an iterative algorithm which converges to an optimal point of the problem, this inter-cluster design based scheme and multivariate compression together improves the performance of compression algorithm. The scheme based on block-ergodic channel fading is discussed in [93], which considers stochastic and instantaneous CSI (channel state information) for joint fronthaul compression design and precoding of C-RAN downlinks. Two different functional splits are studied in this research for the RRHs and BBUs at a physical layer to increase ergodic capacity and reduces the SNR.

The lossless compression scheme is utilized in [94] that is adaptive to the network load. To reduce the load on a fronthaul link only that information are transmitted which are required to reconstruct a control signal at the RRHs. A special constellation coding is employed to represent the QPSK, 16 QAM and 64 QAM modulations in the compression method. This scheme achieves the compression ratio of $33 \%$ at full network load and $7 \%$ at a load of $20 \%$. An efficient baseband signal compression algorithm to reduce the transport data rates is proposed in [95]. It reduces the EVM to 8\% as required by the 3GPP system for 64 QAM signals and to meet the LTE's ACLR (adjacent channel leakage ratio) requirements, the propo-sed scheme provides the ACLR of less than $45 \mathrm{~dB}$ when a relative data rate is higher than $27.78 \%$. It also provides very flexible and implementable solutions to various wireless systems by changing parameters, without changing the architecture, which results in cost reduction.

The uplink and downlink compressions techniques can be suitable solutions for fronthaul networks. The uplink compression is more challenging in fronthaul due to sparsely distribution of UE signals. Point-to-point compression in UL is efficient in reducing the number of transmissions and improving the throughput while spatial compression can be utilized to reduce the SINR. In DL, lossless compression proves promising to enhance the weighted sum and detecting the number of active users to allocate the resources accurately while the block-scaling with quantization techniques are effective in reducing the EVM and SNR at high compression ratios. Therefore, by utilizing efficient compression techniques, the load on capacity-constrained FH can be decreased without increasing the number of fiber resources.

\section{Conclusion}

In this paper, a survey of fronthaul requirements and recent developments in term of architecture, virtualization techniques, transport technologies, and compression techniques are presented. These aspects are surveyed to identify the extensive challenges and solutions that are recently proposed to make the fronthaul a credible technology for commercial deployment of C-RANs in future 5G mobile cellular systems.

It is concluded that more rigorous investigation is required to transform legacy fully centralized C-RAN architecture into partially or hybrid centralization architectures to achieve required throughputs and latencies. Software-defined fronthaul networks can be a promising solution to provide real-time mobile cloud computing services and virtually traffic load management in C-RANs. Novel SDN-based infrastructures and algorithms are still needed to achieve the better traffic management, QoS, self-optimizing, self-configuring, and self-healing operations under different traffic trends. 
On the other hand, cost of the fronthaul link can be reduced by adopting technologies such as CPRI over Ethernet and microwave for short distance communications. Uplink and downlink compression techniques have a high potential to efficiently reduce the fronthaul traffic load and save the fiber resources, as they only require extra compression and decompression modules at the cost of high complexity in the network.

\section{References}

[1] Min Sheng, Weijia Han, Chuan Huang, Jiandong Li, and Shuguang Cui, "Video delivery in heterogeneous CRANs: architectures and strategies," IEEE Wireless Communications, vol. 22, no. 3, pp. 14-21, June 2015. Article (CrossRef Link)

[2] Chih-Lin I, Corbett Rowell, Shuangfeng Han, Zhikun Xu, Gang Li, and Zhengang Pan, "Toward green and soft: a 5G perspective,” IEEE Communications Magazine, vol. 52, no. 2, pp. 66-73, February 2014. Article (CrossRef Link)

[3] Mamta Agarwal, Navrati Saxena, and Abhishek Roy, "Towards connected living: 5G enabled internet of things (IoT),” IETE Technical Review, pp. 1-13, 2018. Article (CrossRef Link)

[4] Serkan Ak, Hazer Inaltekin, and H. Vincent Poor, "A tractable framework for the analysis of dense heterogeneous cellular networks,” IEEE Transactions on Communications, February 2018. Article (CrossRef Link)

[5] Erik G. Larsson, Ove Edfors, Fredrik Tufvesson, and Thomas L. Marzetta, "Massive MIMO for next generation wireless systems," IEEE Communications Magazine, vol. 52, no. 2, February 2014. Article (CrossRef Link)

[6] Volker Jungnickel et al., "The role of small cells, coordinated multipoint, and massive MIMO in 5G,” IEEE Communications Magazine, vol. 52, no. 5, May 2014. Article (CrossRef Link)

[7] Jun-ichi Kani, Jun Terada, Ken-Ichi Suzuki, and Akihiro Otaka, "Solutions for future mobile fronthaul and access-network convergence,” Journal of Lightwave Technology, vol. 35, pp. 527-534, 2017. Article (CrossRef Link)

[8] Jun Wu, Zhifeng Zhang, Yu Hong, and Yonggang Wen, "Cloud Radio Access Network (C-RAN): a primer,” IEEE Networks, vol. 29, pp. 35-41, 2015. Article (CrossRef Link)

[9] Thomas Pfeiffer, "Next generation mobile fronthaul and midhaul architectures," Journal of Optical Communications and Networking, vol. 7, pp. 38-45, 2015. Article (CrossRef Link)

[10] 3GPP TR 36.819, "Coordinated multipoint transmission for LTE physical layer aspects," vol.11.1.0, 2012.

[11] Peter Rost et al., "Benefits and challenges of virtualization in 5G radio access networks," IEEE Communications Magazine, vol. 53, pp. 75-82, 2015. Article (CrossRef Link)

[12] Mugen Peng, Chonggang Wang, Vincent Lau, and H. Vincent Poor, "Fronthaul-constrained cloud radio access networks: insights and challenges," IEEE Wireless Communications, vol. 22, pp. 152-160, 2015. Article (CrossRef Link)

[13] Vinay Suryaprakash, Peter Rost, and Gerhard Fettweis, “Are heterogeneous cloud based radio access networks cost effective?” IEEE Journal of Selected Areas Communications, vol. 33, no. 10, pp. 2239-2251, October 2015. Article (CrossRef Link)

[14] China Mobile, "C-RAN: The road towards green RAN," White Paper, version 2, October 2011.

[15] 3GPP TR 38.801, "Study on new radio access technology - Radio access architecture and interfaces,” Tech. report, version 14.0.0, 2017.

[16] Open Base Station Architecture Initiative (OBSAI) BTS system reference document version 2.0, 2006.

[17] Common Public Radio Interface (CPRI), interface specification, version 7.0, August 2015.

[18] Requirements for Open Radio equipment Interface (ORI), version 4.1.1, 2014.

[19] Antonio de la Oliva, Jose Alberto Hernandez, David Larrabeiti, and Arturo Azcorra, "An overview of the CPRI specification and its application to C-RAN based LTE scenarios," IEEE Communic-ations Magazine, vol. 54, pp. 152-159, 2016. Article (CrossRef Link) 
[20] Jouni Korhonen, "Practical approach to converged FH/BH network architecture and functional partitioning,” IEEE 1914.1 TF, 2016.

[21] Maria Morant, Andres Macho, and Roberto Llorente, "On the suitability of multicore fiber for LTE-advanced MIMO optical fronthaul systems,” Journal of Lightwave Technology, vol. 34, no. 2, pp. 676-682, January 2016. Article (CrossRef Link)

[22] Chih-Lin I, Jinri Huang, Yannan Yuan, and Shijia Ma, “5G RAN architecture: C-RAN with NGFI,” 5G Mobile Communications, Springer, pp 431-455, October 2016. Article (CrossRef Link)

[23] Osvaldo Simeone, Andreas Maeder, Mugen Peng, Onur Sahin, and Wei Yu, "Cloud radio access network: Virtualizing wireless access for dense heterogeneous systems," Journal of Communications and Networks, vol. 18, no. 2, pp. 135-149, April 2016. Article (CrossRef Link)

[24] Xiongbin Rao and Vincent K. N. Lau, "Distributed fronthaul compression and joint signal recovery in cloud-RAN,” IEEE Transactions on Signal Processing, vol. 63, no. 4, pp. 1056-1065, February 2015. Article (CrossRef Link)

[25] Small cell virtualization functional splits and use cases, Small Cell Forum, Tech. rep., June 2015.

[26] Dario Sabella et al., "RAN as a service: challenges of designing a flexible RAN architecture in a cloud-based heterogeneous mobile network," in Proc. of Future Network Summit, pp. 1-8, July 2013.

[27] Aleksandra Checko, Andrijana Popovska Avramova, Michael Stubert Berger, and Henrik Lehrmann Christiansen, "Evaluating CRAN fronthaul functional splits in terms of network level energy and cost savings,” Journal of Communications and Networks, vol. 18, no. 2, April 2016. Article (CrossRef Link)

[28] Ian F.Akyildiz, PuWang, and Shih-ChunLin, "SoftAir: a software defined networking architecture for 5G wireless systems,” Computer Networks, vol. 85, pp. 1-18, 2015. Article (CrossRef Link)

[29] Jens Bartelt, Peter Rost, Dirk Wubben, Johannes Lessmann, Bruno Melis, and Gerhard Fettweis, "Fronthaul and backhaul requirements of flexibly centralized radio access networks," IEEE Wireless Communications, October 2015. Article (CrossRef Link)

[30] NTT DoCoMo, “5G radio access: requirements, concept and technologies,” Tech. Rep., July 2014.

[31] Sangkyu Park, Chan-Byoung Chae, and Saewoong Bahk, "Large-scale antenna operation in heterogeneous cloud radio access networks: a partial centralization approach,” IEEE Wireless Communications, vol. 22, no. 3, pp. 32-40, July 2015. Article (CrossRef Link)

[32] Mugen Peng, Shi Yan, Kecheng Zhang, and Chonggang Wang, "Fog computing based radio access networks: Issues and challenges,” IEEE Network, vol. 30, no. 4, pp. 46-53, 2016. Article (CrossRef Link)

[33] 5G Radio Access: Requirements, Concept and Technologies, DOCOMO, 5G Paper, 2014.

[34] 5G Vision, Samsung Electronics Co., Ltd., 2015.

[35] Y. Lin, L. Shao, Z. Zhu, Q. Wang, and R. K. Sabhikhi "Wireless network cloud: architecture and system requirements,” IBM Journal of Research and Development, vol. 54, no. 1, pp. 1-4, 2010. Article (CrossRef Link)

[36] ZTE green technology innovations, white paper, Shenzhen, China, Tech. Rep., 2011.

[37] Bernd Haberland et al., "Radio base stations in the cloud,” Bell Labs Technical Journal, vol. 18, no. 1, pp. 129-152, 2013. Article (CrossRef Link)

[38] J. Segel et al., "LightRadio portfolio: white paper 3,” France, Tech. Rep., 2011.

[39] Nokia Networks, "Liquid radio making radio networks active, adaptive and aware,” Tech. Rep., 2014.

[40] H. Guan, T. Kolding, and P. Merz, “Discovery of cloud-RAN,” Nokia siemens networks, Tech. Rep., April 2010.

[41] Ericsson mobility report: on the pulse of the networked society, Ericsson, 2015.

[42] OpenAirInterface, [Online]. Available: Article (CrossRef Link)

[43] E-BLINK, wireless fronthaul technology, [Online]. Available: Article (CrossRef Link)

[44] Clark Chen, "C-RAN: The road towards green radio access network," China Mobile Research Institute, August 2012.

[45] Rethink Wireless, “Korea telecom plans world’s first commercial cloud-RAN,” IBM Research Gabriel, December 2011.

[46] Enrique Hernandez-Valencia, Steven Izzo, and Beth Polonsky, "How will NFV/SDN transform service provider OPEX,” IEEE Networks, vol. 29, no. 3, pp. 79-108, 2015. Article (CrossRef Link) 
[47] Fei Hu, Qi Hao, and Ke Bao, “A survey on software-defined network and OpenFlow: From concept to implementation,” IEEE Communications Surveys \& Tutorials, vol. 16, no. 4, pp. 2181-2206, 2014. Article (CrossRef Link)

[48] Jan Medved, Robert Varga, Anton Tkacik, and Ken Gray, “OpenDaylight: towards a model-driven SDN controller architecture," in Proc. of IEEE 15th International Symposium on World of Wireless, Mobile and Multimedia Networks (WoWMoM), vol. 5, no. 2, pp.1-6, 19-19 June 2014. Article (CrossRef Link)

[49] Mustafa Y. Arslan, Karthikeyan Sundaresan, and Sampath Rangarajan, "Software-defined networking in cellular radio access networks: potential and challenges," IEEE Communications Magazine, vol. 53, no. 1, pp. 150-156, January 2015. Article (CrossRef Link)

[50] Karthikeyan Sundaresan, Mustafa Y. Arslan, Shailendra Singh, Sampath Rangarajan, and Srikanth V. Krishnamurthy, "FluidNet: a flexible cloud based radio access network for small cells," IEE/ACM Transactions on Networking, vol. 24, no. 2, April 2016. Article (CrossRef Link)

[51] Mohamed Selim, Ahmed E. Kamal, Khaled Elsayed, Heba M. Abdel-Atty, and Mohammed Alnuem, "Fronthaul cell outage compensation for 5G networks," IEEE Communications Magazine, August 2016. Article (CrossRef Link)

[52] Sergio Gonzalez et al., "5G-Crosshaul: an SDN/NFV control and data plane architecture for the 5G integrated fronthaul/backhaul," Transactions on Emerging Telecommunications Technologies, 2016. Article (CrossRef Link)

[53] Avishek Nag, Yi Zhang, Luiz A. DaSilva, Linda Doyle, and Marco Ruffini, "Integrating wireless BBUs with optical OFDM flexible-Grid transponders in a C-RAN architecture,” in Proc. of OFC, 2017. Article (CrossRef Link)

[54] Jiawei Zhang et al., "Baseband unit cloud interconnection enabled by flexible grid optical networks with software defined elasticity,” IEEE Communications Magazine, vol. 53, no. 9, pp. 90-98, September 2016. Article (CrossRef Link)

[55] Jiawei Zhang et al., "Experimental demonstration of fronthaul flexibility for enhanced CoMP service in 5G radio and optical access networks,” Optics Express, vol. 25, no. 18, pp. 21247-21258, 2017. Article (CrossRef Link)

[56] Jiawei Zhang et al., "Reconfigurable optical mobile fronthaul networks for coordinated multipoint transmission and reception in the 5G,” IEEE/OSA Journal of Optical Communications and Networking, vol. 9, no. 6, pp. 489-497, 2017. Article (CrossRef Link)

[57] Jiawei Zhang et al., "Energy efficient baseband unit aggregation in cloud radio and optical access networks,” IEEE/OSA Journal of Optical Communications and Networking, vol. 8, no. 11, pp. 893-901, 2016. Article (CrossRef Link)

[58] Scenarios and requirements for small cell enhancements for E-UTRA and E-UTRAN, version 12.1.0, Sophia-Antipolis Cedex, France, TR 36.932, March 2013.

[59] Zere Ghebretensae et al., "Transmission solutions and architectures for heterogeneous networks built as C-RANs,” in Proc. of 7th International ICST Conference CHINACOM, pp. 748-752, 2012. Article (CrossRef Link)

[60] Humair Raza, “A brief survey of radio access network backhaul evolution: Part I," IEEE Communication Magazine, vol. 49, no. 6, pp. 164-171, June 2011. Article (CrossRef Link)

[61] IEEE P802.1Qbu-Bridges and bridged networks-Amendment: Enhancements for frame preemption, 2015.

[62] IEEE P802.1Qbv-Bridges and bridged networks-Amendment: Enhancements for scheduled traffic, 2015.

[63] Tao Wan and Peter Ashwood-Smith, “A performance study of CPRI over Ethernet with IEEE 802.1Qbu and 802.1Qbv enhancements,” in Proc. of IEEE Global Communications Conference (GLOBECOM), pp. 1-6, 6-10 December 2015. Article (CrossRef Link)

[64] Tao Wan and Peter Ashwood-Smith, “A performance study of CPRI over Ethernet," white paper, 2015.

[65] Divya Chitimalla, Koteswararao Kondepu, Luca Valcarenghi, Massimo Tornatore, and Biswanath Mukherjee, "5G fronthaul-latency and jitter studies of CPRI over Ethernet," Journal of Optical Communications and Networking, vol. 9, pp. 72-182, 2017. Article (CrossRef Link)

[66] Standard for Radio over Ethernet encapsulations and mappings, IEEE P1914.3, 2017.

[67] Standard for local and metropolitan area networks-Time-sensitive networking for fronthaul, IEEE P802.1CM, 2017. 
[68] Siyu Zhou, Xiang Liu, Frank Effenberger, and Jonathan Chao, "Low-Latency high-efficiency mobile fronthaul with TDM-PON (Mobile-PON)," Journal of Optical Communications and Networking, vol. 10, no. 1, January 2018. Article (CrossRef Link)

[69] P. Parolari et al., "Self-tuning transmitter for fiber-to-the-antenna PON networks, optical switching and networking," Optical Switching and Networking, vol. 14, no. 1, pp. 25-31, August 2014. Article (CrossRef Link)

[70] Yu Nakayama, Daisuke Hisano, Takahiro Kubo, Youichi Fukada, Jun Terada, and Akihiro Otaka, "Low-latency routing scheme for a fronthaul bridged network," Journal of Optical Communications and Networking, vol. 10, no 1, pp. 14-23, 2018. Article (CrossRef Link)

[71] Philippe Chanclou et al., "Optical fiber solution for mobile fronthaul to achieve cloud radio access network,” in Proc. of Future Network \& Mobile Summit 2013 Conference Proceedings, Lisboa, pp.1-11, July 2013.

[72] Filippo Ponzini, Luca Giorgi, Alberto Bianchi, and Roberto Sabella, "Centralized radio access networks over wavelength-division multiplexing: A plug and-play implementation,” IEEE Communication Magazine, vol. 51, no. 9, September 2013. Article (CrossRef Link)

[73] Wang Huitao and Zhao Yong, “C-RAN bearer network solution,” ZTE, Tech. rep., November 2011.

[74] Ming Zhu, Fan Li, Feng Lu, Jianjun Yu, Charles Su, Gordon Gu, and Gee-Kung Chang, "Wavelength resource sharing in bidirectional optical mobile fronthaul," Journal of Lightwave Technology, vol. 33, no. 15, pp. 3182-3188, August 2015. Article (CrossRef Link)

[75] Zere Ghebretensae et al., "Transmission solutions and architectures for heterogeneous networks built as C-RANs," in Proc. of 7th International ICST Conference on Communications and Networking in China (CHINACOM), pp. 748-752, 2012. Article (CrossRef Link)

[76] Jie Hyun Lee, Seung-Hyun Cho, Kyung Hwan Doo, Seung-Il Myong, Jong Hyun Lee, and Sang Soo Lee, "CPRI transceiver for mobile front-haul based on wavelength division multiplexing," in Proc. of International Conference on ICT Convergence (ICTC), pp. 581-582, 2012. Article (CrossRef Link)

[77] Bo Liu, Xiangjun Xin, Lijia Zhang, and Jianjun Yu, “109.92-Gb/s WDM-OFDMA Uni-PON with dynamic resource allocation and variable rate access," Optical Society of America, Optics Express, vol. 20, no. 10, May 2012. Article (CrossRef Link)

[78] Maria Morant, Andres Macho, and Roberto Llorente, “On the suitability of multicore fiber for LTE-Advanced MIMO optical fronthaul systems,” Journal of Lightwave Technology, vol. 34, no. 2, pp. 676-682, January 2016. Article (CrossRef Link)

[79] Lin Cheng, Ming Zhu, Malik Muhammad Usman Gul, Xiaoli Ma, and Gee-Kung Chang, "Adaptive photonics-aided coordinated multipoint transmissions for next-generation mobile fronthaul,” Journal of Lightwave Technology, vol. 32, no. 10, pp. 1907-1914, May 2014. Article (CrossRef Link)

[80] Cheng Liu, Liang Zhang, Ming Zhu, Jing Wang, Lin Cheng, and Gee-Kung Chang, “A novel multi-service small-cell cloud radio access network for mobile backhaul and computing based on radio-over-fiber technologies,” Journal of Lightwave Technology, vol. 31, no. 17, pp. 2869-2875, September 2013. Article (CrossRef Link)

[81] National Instruments, White Paper, March 2017. [Online]. Available: Article (CrossRef Link)

[82] Anritus, [Online]. Available: Article (CrossRef Link)

[83] Checko Aleksandra et al., "OTN transport of baseband radio serial protocols in C-RAN architecture for mobile network applications,” MTI Mobile and Altera, Tech. rep., March 2014.

[84] Altera: SoftSilicon OTN Processors, [Online]. Available: Article (CrossRef Link)

[85] 3GPP TS 36.104 Evolved Universal Terrestrial Radio Access (E-UTRA); Base Station (BS) radio transmission and reception, version 12.0.0. July 2013.

[86] Seongah Jeong, Osvaldo Simeone, Alexander Haimovich, and Joonhyuk Kang, “Optimal fronthaul quantization for cloud radio positioning,” IEEE Transactions on Vehicular Technology, May 2015. Article (CrossRef Link) 
[87] Xiongbin Rao and Vincent K. N. Lau, "Distributed fronthaul compression and joint signal recovery in C-RAN,” IEEE Transactions on Signal Processing, vol. 63, no. 4, pp. 1056-1065, February 2015. Article (CrossRef Link)

[88] Seok-Hwan Park, Osvaldo Simeone, Onur Sahin, and Shlomo Shamai, "Multihop backhaul compression for the uplink of cloud radio access networks," IEEE international symposium on Information theory (ISIT), pp. 2704-2708, June 2014. Article (CrossRef Link)

[89] Liang Liu, Suzhi Bi, and Rui Zhang, "Joint power control and fronthaul rate allocation for throughput maximization in OFDMA-based cloud radio access network,” IEEE Transactions on Communications, vol. 63, no. 11, November 2015. Article (CrossRef Link)

[90] Liang Liu and Rui Zhang, “Optimized uplink transmission in multi-antenna C-RAN with spatial compression and forward," IEEE Transactions on Signal Processing, vol. 63, no. 19, pp. 5083-5095, October 2015. Article (CrossRef Link)

[91] I. D. Technology, “Compression IP for wireless infrastructure applications,” Product brief., July 2013.

[92] Seok-Hwan Park, Osvaldo Simeone, Onur Sahin, and Shlomo Shamai, "Inter-cluster design of precoding and fronthaul compression for cloud radio access networks," IEEE Wireless Communication Letter, vol. 3, no. 4, pp. 369-372, April 2014. Article (CrossRef Link)

[93] Jinkyu Kang, Osvaldo Simeone, Joonhyuk Kang, and Shlomo Shamai, "Fronthaul compression and precoding design for C-RANs over Ergodic fading channels," IEEE Transactions on Vehicular Technology, August 2015. Article (CrossRef Link)

[94] Seok-Hwan Park, Osvaldo Simeone, Onur Sahin, and Shlomo Shamai, "Robust and efficient distributed compression for cloud radio access networks," IEEE Transactions on Vehicular Technology, vol. 62, no. 2, pp. 692-703, February 2013. Article (CrossRef Link)

[95] Dragan Samardzija, John Pastalan, Michael MacDonald, Susan Walker, and Reinaldo Valenzuela, "Compressed transport of baseband signals in radio access networks," IEEE Transactions on Wireless Communications, vol. 11, no. 9, pp. 3216-3225, September 2012. Article (CrossRef Link) 


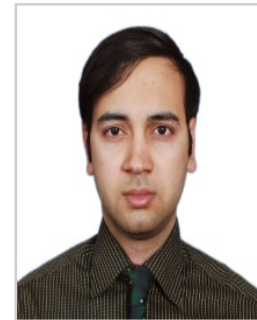

MUHAMMAD WAQAR received a B.S. degree in Computer Engineering and M.S. degree in Electrical Engineering from COMSATS University, Islamabad, Pakistan, in 2011 and 2015, respectively. He is currently pursuing a Ph.D. degree at Sejong University, Seoul, South Korea, and is researching 5G fronthaul solutions. His research interests include 5G networks, optical networks and SDNs.

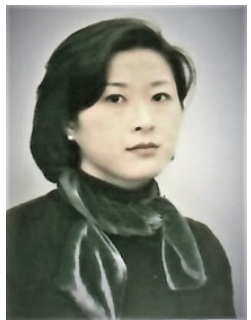

AJUNG KIM received a B.S. degree in Physics from Seoul National University, Korea, in 1988, and M.S. degree and Ph.D. degree majoring in Information Theory from Northwestern University, Evanston, USA. She had also worked as a post-doctoral research fellow and research associate at Northwestern University. She also worked with Samsung Electronics, and with the School of Engineering and Applied Science at Harvard University as an exchange professor. Since 2003, she has been a professor with the Department of Optical Engineering at Sejong University, Seoul, Korea. Her research interests include network communications and optical networks.

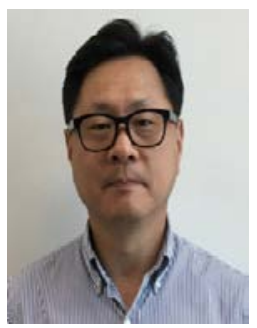

PETER K. CHO received a B.S. degree in Computer Science from Seogang University, Korea, in 1985 and an M.S. degree from KAIST, Korea, in 2001. He worked as a project manager at Samsung Electronics and as a product manager at Cisco Systems. He has been CEO at Actus Networks Inc., and Vice President at HFR Inc., both of which are Korea's representative vendors of wired and wireless network equipment. His research interests include $5 \mathrm{G}$ fronthaul networks, SDNs and carrier Ethernet. 